PROCEEDINGS OF THE

AMERICAN MATHEMATICAL SOCIETY

Volume 124, Number 12, December 1996, Pages 3789-3795

S 0002-9939(96)03566-6

\title{
ALMOST EVERYWHERE CONVERGENCE OF LACUNARY PARTIAL SUMS OF VILENKIN-FOURIER SERIES
}

\author{
WO-SANG YOUNG
}

(Communicated by J. Marshall Ash)

\begin{abstract}
We prove that if $f \in L^{p}, p>1$, and $\left\{n_{k}\right\}$ is any lacunary sequence of positive integers, then the sequence of $n_{k}$ th partial sums of Vilenkin-Fourier series of $f$ converges almost everywhere to $f$.
\end{abstract}

\section{INTRODUCTION}

Let $G=\prod_{i=0}^{\infty} Z_{p_{i}}$ be the direct product of cyclic groups of order $p_{i}$, where $\left\{p_{i}\right\}_{i \geq 0}$ is a sequence of integers with $p_{i} \geq 2$. Let $\mu$ be the Haar measure on $G$ normalized by $\mu(G)=1$. For $x=\left\{x_{k}\right\} \in G$, let $\phi_{k}(x)=\exp \left(2 \pi i x_{k} / p_{k}\right), k=0,1,2, \ldots$. The Vilenkin system $\left\{\chi_{n}\right\}$ is the set of all finite products of $\left\{\phi_{k}\right\}$, and is enumerated in the following manner. Let $m_{0}=1, m_{k}=\prod_{i=0}^{k-1} p_{i}, k=1,2, \ldots$ We express each nonnegative integer $n$ as a finite sum in the form $n=\sum_{k=0}^{\infty} \alpha_{k} m_{k}$, where $0 \leq \alpha_{k}<p_{k}$, and define $\chi_{n}=\prod_{k=0}^{\infty} \phi_{k}^{\alpha_{k}}$. The functions $\left\{\chi_{n}\right\}$ are the characters of $G$, and they form a complete orthonormal system on $G$. If $p_{i}=2, i=0,1,2, \ldots$, then $\left\{\phi_{k}\right\}$ are the Rademacher functions and $\left\{\chi_{n}\right\}$ are the Walsh functions. In this paper there is no restriction on the orders $\left\{p_{i}\right\}$.

We consider Fourier series with respect to $\left\{\chi_{n}\right\}$. For $f \in L^{1}$, let $\widehat{f}(j)=$ $\int_{G} f(t) \overline{\chi_{j}(t)} d \mu(t), j=0,1,2, \ldots$, and $S_{n} f=\sum_{j=0}^{n-1} \widehat{f}(j) \chi_{j}, n=1,2, \ldots$ Not much is known about the almost everywhere convergence of partial sums when there is no restriction on the orders $\left\{p_{i}\right\}$. We have the following result.

Theorem. Let $1<p<\infty$ and $\left\{n_{k}\right\}_{k \geq 1}$ be a lacunary sequence of positive integers, i.e., there is $q>1$ such that $n_{k+1} / n_{k} \geq q, k=1,2, \ldots$ Then there is a constant $C_{p}$ such that

$$
\left\|\sup _{k}\left|S_{n_{k}} f\right|\right\|_{p} \leq C_{p}\|f\|_{p}, \quad f \in L^{p} .
$$

It follows that $\lim _{k \rightarrow \infty} S_{n_{k}} f(x)=f(x)$ a.e. for all $f \in L^{p}$.

Received by the editors March 8, 1995 and, in revised form, June 15, 1995.

1991 Mathematics Subject Classification. Primary 42C10; Secondary 42B25, 43A75.

(c)1996 American Mathematical Society 
In the proof that follows, $C$ and $C_{p}$ will denote absolute constants which may vary from line to line.

\section{Proof of the Theorem}

We first prove (1.1). For $k=0,1, \ldots$, let $L_{k}$ be the positive integer such that $2^{L_{k}} \leq p_{k}<2^{L_{k}+1}$. Since every lacunary sequence can be decomposed into a finite number of lacunary subsequences with ratio $q=2$, we may assume, by adding more terms to the sequence if necessary, that $\left\{n_{j}\right\}$ can be relabelled $\left\{n_{k, \ell}\right\}_{k=0,1, \ldots, \ell=0,1, \ldots, L_{k}}$, such that for $k=0,1, \ldots, 2^{\ell} m_{k} \leq n_{k, \ell}<$ $2^{\ell+1} m_{k} \quad$ if $\ell=0,1, \ldots, L_{k}-1$, and $2^{L_{k}} m_{k} \leq n_{k, L_{k}}<m_{k+1}$. (There is no $n_{k, L_{k}}$ term if $2^{L_{k}} m_{k}=m_{k+1}$.) Also, it is sufficient to show that there is a constant $C_{p}$ such that

$$
\left\|\sup _{\substack{\ell=0, \ldots, L_{k} \\ k=0, \ldots, N-1}}\left|S_{n_{k, \ell}} f\right|\right\|_{p} \leq C_{p}\|f\|_{p}
$$

for all $f \in L^{p}, N=1,2, \ldots$.

Let $f_{k}=S_{m_{k+1}} f-S_{m_{k}} f, k=0,1, \ldots$, and $f_{-1}=S_{1} f$. We observe that

$$
\sup _{\substack{\ell=0, \ldots, L_{k} \\ k=0, \ldots, N-1}}\left|S_{n_{k, \ell}} f\right| \leq \sup _{\substack{\ell=0, \ldots, L_{k} \\ k=0, \ldots, N-1}}\left|S_{n_{k, \ell}} f_{k}\right|+\sup _{k=0, \ldots, N-1}\left|S_{m_{k}} f\right| .
$$

Since $\left\{S_{m_{k}} f\right\}$ is a martingale (see, e.g., [5]), it follows from Doob's inequality $\left(\left\|\sup _{k \geq 0}\left|S_{m_{k}} f\right|\right\|_{p} \leq C_{p}\|f\|_{p}, f \in L^{p}\right)$ that (2.1) will be proved if we have

$$
\left\|\sup _{\substack{\ell=0, \ldots, L_{k} \\ k=0, \ldots, N-1}}\left|S_{n_{k, \ell}} f_{k}\right|\right\|_{p} \leq C_{p}\|f\|_{p},
$$

for all $f \in L^{p}, N=1,2, \ldots$.

Now,

$$
\begin{aligned}
\sup _{\substack{\ell=0, \ldots, L_{k} \\
k=0, \ldots, N-1}}\left|S_{n_{k, \ell}} f_{k}\right| \leq & \left(\sum_{k=0}^{N-1}\left|S_{n_{k, 0}} f_{k}\right|^{2}\right)^{1 / 2}+\sup _{\substack{\ell=1, \ldots, L_{k}-2 \\
k=0, \ldots, N-1}}\left|S_{n_{k, \ell}} f_{k}\right| \\
& +\left(\sum_{k=0}^{N-1}\left|S_{n_{k, L_{k}-1}} f_{k}\right|^{2}\right)^{1 / 2}+\left(\sum_{k=0}^{N-1}\left|S_{n_{k, L_{k}}} f_{k}\right|^{2}\right)^{1 / 2} .
\end{aligned}
$$

For each of $\ell=0, L_{k}-1$ and $L_{k}$, we apply [5, Theorem 2] and Burkholder's result for martingales [1, Theorem 3.2] to get

$$
\begin{aligned}
\left\|\left(\sum_{k=0}^{N-1}\left|S_{n_{k, \ell}} f_{k}\right|^{2}\right)^{1 / 2}\right\|_{p} & \leq C_{p}\left\|\left(\sum_{k=0}^{N-1}\left|f_{k}\right|^{2}\right)^{1 / 2}\right\|_{p} \\
& \leq C_{p}\|f\|_{p} .
\end{aligned}
$$

To prove (2.2) it remains to show

$$
\left\|\sup _{\substack{\ell=1, \ldots, L_{k}-2 \\ k=0, \ldots, N-1}}\left|S_{n_{k, \ell}} f_{k}\right|\right\|_{p} \leq C_{p}\|f\|_{p}
$$

for all $f \in L^{p}, N=1,2, \ldots$. 
We shall use the following operators. Let $k=0,1, \ldots$ If $L_{k}>2$, define, for $\ell=1, \ldots, L_{k}-2$, the sequence $\left\{a_{k, \ell}(n)\right\}_{n \geq 0}$ by

$$
a_{k, \ell}(n)= \begin{cases}1 & \text { if } 2^{\ell} m_{k} \leq n<2^{\ell+1} m_{k}, \\ \frac{j}{2^{\ell-1}} & \text { if }\left(2^{\ell-1}+j\right) m_{k} \leq n<\left(2^{\ell-1}+j+1\right) m_{k}, \\ 1-\frac{j+1}{2^{\ell-1}} & \text { if }\left(2^{\ell+1}+j\right) m_{k} \leq n<\left(2^{\ell+1}+j+1\right) m_{k}, \\ & j=0,1, \ldots, 2^{\ell-1}-1, \\ 0 & \text { otherwise },\end{cases}
$$

and set

$$
A_{k, \ell} f=\sum_{n=0}^{\infty} a_{k, \ell}(n) \widehat{f}(n) \chi_{n} .
$$

It is proved in [6, pp. 665-666] that

$$
\left\|\left(\sum_{k=0}^{N-1} \sum_{\ell=1}^{L_{k}-2}\left|A_{k, \ell} f\right|^{2}\right)^{1 / 2}\right\|_{p} \leq C_{p}\|f\|_{p}, \quad f \in L^{p}, \quad N=1,2, \ldots .
$$

(We interpret a sum $\sum_{j=k}^{\ell}$ with $\ell<k$ as zero.)

The second operator is defined as follows. Let $k=0,1, \ldots$ If $L_{k} \geq 2$, define, for $\ell=1, \ldots, L_{k}-1$, the sequence $\left\{b_{k, \ell}(n)\right\}_{n \geq 0}$ by

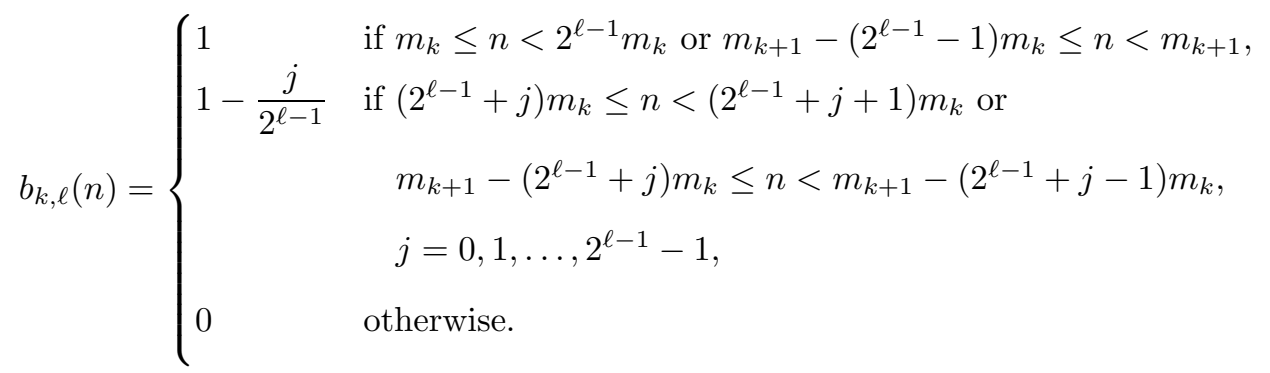

For $f \in L^{1}$, set

$$
B_{k, \ell} f=\sum_{n=0}^{\infty} b_{k, \ell}(n) \widehat{f}(n) \chi_{n}
$$

To estimate $B_{k, \ell} f$, we need the following definitions. Let $\left\{G_{k}\right\}$ be the sequence of subgroups of $G$ defined by

$$
G_{0}=G, \quad G_{k}=\prod_{i=0}^{k-1}\{0\} \times \prod_{i=k}^{\infty} Z_{p_{i}}, \quad k=1,2, \ldots
$$

We shall identify $G$ with the unit interval $(0,1)$ by associating with each $\left\{x_{i}\right\} \in$ $G, \quad 0 \leq x_{i}<p_{i}$, the point $\sum_{i=0}^{\infty} x_{i} m_{i+1}^{-1} \in(0,1)$. If we disregard the countable set of $p_{i}$-rationals, this mapping is one-to-one, onto and measure preserving. On the 
interval $(0,1)$, cosets of $G_{k}$ are intervals of the form $\left(j m_{k}^{-1},(j+1) m_{k}^{-1}\right), j=$ $0,1, \ldots, m_{k}-1$. A set $I$ is called a generalized interval if $I \subset x+G_{k}$ for some $x \in G, k=0,1, \ldots, I$ is a union of cosets of $G_{k+1}$, and $I$ is an interval if we consider $x+G_{k}$ as a circle.

Let

$$
M f(x)=\sup _{\substack{x \in I \\ I \text { generalized } \\ \text { interval }}} \frac{1}{\mu(I)} \int_{I}|f| d \mu
$$

be the Hardy-Littlewood maximal function for the Vilenkin system. The following pointwise estimate will be obtained in $\S 3$.

Lemma. There is a constant $C$ such that

$$
\sup _{\substack{\ell=1, \ldots, L_{k}-1 \\ k=0,1, \ldots}}\left|B_{k, \ell} f(x)\right| \leq C M f(x), \quad f \in L^{1}, x \in G .
$$

To estimate $S_{n_{k, \ell}} f_{k}, \quad \ell=1, \ldots, L_{k}-2, \quad k=0,1, \ldots$, we shall use the part of $b_{k, \ell}(n)$ with $n \in\left[m_{k}, 2^{\ell} m_{k}\right)$. In order to get rid of the remaining part of $b_{k, \ell}(n)$, we define

$$
H^{N} f=\sum_{k=0}^{N-1}\left(S_{2^{L_{k}-1} m_{k}} f-S_{m_{k}} f\right) .
$$

Since $H^{N} f=S_{m_{N}} f-\sum_{k=0}^{N-1}\left(S_{m_{k+1}} f-S_{2^{L_{k}-1} m_{k}} f\right)$, and it is proved in [4, Theorem $1^{*}$ that

$$
\left\|\sum_{k=0}^{N-1}\left(S_{m_{k+1}} f-S_{2^{L_{k}-1} m_{k}} f\right)\right\|_{p} \leq C_{p}\|f\|_{p}
$$

for all $f \in L^{p}, N=1,2, \ldots$, we have

$$
\left\|H^{N} f\right\|_{p} \leq C_{p}\|f\|_{p}, \quad f \in L^{p}, \quad N=1,2, \ldots .
$$

(See also [2].)

We are now ready to prove (2.3). We observe that for $k=0, \ldots, N-1$, $\ell=1, \ldots, L_{k}-2$,

$$
S_{n_{k, \ell}} f_{k}=B_{k, \ell}\left(H^{N} f\right)+S_{n_{k, \ell}}\left(A_{k, \ell} f\right) .
$$

Hence

$$
\sup _{\substack{\ell=1, \ldots, L_{k}-2 \\ k=0, \ldots, N-1}}\left|S_{n_{k, \ell}} f_{k}\right| \leq \sup _{\substack{\ell=1, \ldots, L_{k}-2 \\ k=0, \ldots, N-1}} B_{k, \ell}\left(H^{N} f\right)+\left(\sum_{k=0}^{N-1} \sum_{\ell=1}^{L_{k}-2}\left|S_{n_{k, \ell}}\left(A_{k, \ell} f\right)\right|^{2}\right)^{1 / 2}
$$

To estimate the first term on the right, we apply the lemma, the fact that the Hardy-Littlewood maximal operator $M$ is bounded in $L^{p}$ (see [3]) and (2.5). We have

$$
\begin{aligned}
\left\|\sup _{\substack{\ell=1, \ldots, L_{k}-2 \\
k=0, \ldots, N-1}}\left|B_{k, \ell}\left(H^{N} f\right)\right|\right\|_{p} & \leq C\left\|M\left(H^{N} f\right)\right\|_{p} \\
& \leq C_{p}\left\|H^{N} f\right\|_{p} \leq C_{p}\|f\|_{p}
\end{aligned}
$$


for all $f \in L^{p}, N=1,2, \ldots$. For the last term in (2.6) we use [5, Theorem 2] and (2.4). We obtain

$$
\begin{aligned}
\left\|\left(\sum_{k=0}^{N-1} \sum_{\ell=1}^{L_{k}-2}\left|S_{n_{k, \ell}}\left(A_{k, \ell} f\right)\right|^{2}\right)^{1 / 2}\right\|_{p} & \leq C_{p}\left\|\left(\sum_{k=0}^{N-1} \sum_{\ell=1}^{L_{k}-2}\left|A_{k, \ell} f\right|^{2}\right)^{1 / 2}\right\|_{p} \\
& \leq C_{p}\|f\|_{p}
\end{aligned}
$$

for all $f \in L^{p}, N=1,2, \ldots$. This proves (2.3). The proof of (1.1) will be complete once we prove the lemma.

Finally, if $f \in L^{p}, \lim _{k \rightarrow \infty}\left\|S_{m_{k}} f-f\right\|_{p}=0$ since $S_{m_{k}} f$ is the average of $f$ over the cosets of $G_{k}$. As a consequence of this and (1.1), we have $\lim _{k \rightarrow \infty} S_{n_{k}} f(x)=f(x)$ a.e.

\section{Proof of the Lemma}

We shall use the following notation. For each generalized interval $I$, we define the generalized interval $3 I$ as follows. If $I=G$, let $3 I=G$. Suppose $I$ is a proper subset of $x+G_{k}, x \in G, k=0,1,2, \ldots$, and is the union of cosets of $G_{k+1}$. If $\mu(I) \geq \mu\left(G_{k}\right) / 3$, let $3 I=x+G_{k}$. If $\mu(I)<\mu\left(G_{k}\right) / 3$, consider $x+G_{k}$ as a circle and define $3 I$ to be the interval in this circle which has the same center as $I$ and has measure $\mu(3 I)=3 \mu(I)$. For all cases, we have $\mu(3 I) \leq 3 \mu(I)$.

For $k=0,1, \ldots, \ell=1, \ldots, L_{k}-1$ and $f \in L^{1}$, we have

$$
B_{k, \ell} f(x)=\int_{G} f(t)\left[\sum_{n=0}^{\infty} b_{k, \ell}(n) \chi_{n}(x-t)\right] d \mu(t) .
$$

Since $b_{k, \ell}(n)$ vanishes for $n \notin\left[m_{k}, m_{k+1}\right)$ and is constant for $n \in\left[\alpha m_{k}\right.$, $\left.(\alpha+1) m_{k}\right), \alpha=0,1, \ldots$, we get

$$
\begin{aligned}
\sum_{n=0}^{\infty} b_{k, \ell}(n) \chi_{n} & =\sum_{\alpha=1}^{p_{k}-1} b_{k, \ell}\left(\alpha m_{k}\right) \sum_{n=\alpha m_{k}}^{(\alpha+1) m_{k}-1} \chi_{n} \\
& =\sum_{\alpha=1}^{p_{k}-1} b_{k, \ell}\left(\alpha m_{k}\right) \phi_{k}^{\alpha} D_{m_{k}},
\end{aligned}
$$

where $D_{n}=\sum_{j=0}^{n-1} \chi_{j}, n=1,2, \ldots$, denotes the $n^{\text {th }}$ Dirichlet kernel. Since $D_{m_{k}}=$ $\mu\left(G_{k}\right)^{-1} \chi_{G_{k}}$, we have

$$
B_{k, \ell} f(x)=\frac{1}{\mu\left(G_{k}\right)} \int_{x+G_{k}} f(t) M_{k, \ell}(x-t) d \mu(t),
$$

where

$$
\begin{aligned}
M_{k, \ell} & =\sum_{\alpha=1}^{p_{k}-1} b_{k, \ell}\left(\alpha m_{k}\right) \phi_{k}^{\alpha} \\
& =\sum_{\alpha=1}^{2^{\ell}-1} b_{k, \ell}\left(\alpha m_{k}\right) \phi_{k}^{\alpha}+\phi_{k}^{0}+\sum_{\alpha=-\left(2^{\ell}-1\right)}^{-1} b_{k, \ell}\left(\left(p_{k}+\alpha\right) m_{k}\right) \phi_{k}^{\alpha}-\phi_{k}^{0} .
\end{aligned}
$$


Let

$$
D_{k, j}=\sum_{\alpha=-j}^{j} \phi_{k}^{\alpha}, \quad j=0,1, \ldots, 2^{L_{k}-1}-1,
$$

and

$$
K_{k, n}=\frac{1}{n} \sum_{j=0}^{n-1} D_{k, j}, \quad n=1, \ldots, 2^{L_{k}-1}
$$

Then

$$
\begin{aligned}
M_{k, \ell} & =\frac{1}{2^{\ell-1}}\left(D_{k, 2^{\ell-1}}+D_{k, 2^{\ell-1}+1}+\cdots+D_{k, 2^{\ell}-1}\right)-\phi_{k}^{0} \\
& =2 K_{k, 2^{\ell}}-K_{k, 2^{\ell-1}}-\phi_{k}^{0} .
\end{aligned}
$$

For $n=1, \ldots, 2^{L_{k}-1}$, let

$$
\sigma_{k, n} f(x)=\frac{1}{\mu\left(G_{k}\right)} \int_{x+G_{k}} f(t) K_{k, n}(x-t) d \mu(t) .
$$

From (3.1) and (3.2) we obtain

$$
\left|B_{k, \ell} f(x)\right| \leq 2\left|\sigma_{k, 2^{\ell}} f(x)\right|+\left|\sigma_{k, 2^{\ell-1}} f(x)\right|+M f(x) .
$$

The lemma will be proved if we show

$$
\left|\sigma_{k, n} f(x)\right| \leq C M f(x),
$$

for all $k=0,1, \ldots, n=1, \ldots, 2^{L_{k}-1}, \quad x \in G$.

By a direct computation we have

$$
K_{k, n}(x)= \begin{cases}\frac{1}{n}\left(\sin ^{2} \frac{\pi n x_{k}}{p_{k}} / \sin ^{2} \frac{\pi x_{k}}{p_{k}}\right) & \text { if } x_{k} \neq 0 \\ n & \text { if } x_{k}=0\end{cases}
$$

and hence

$$
0 \leq K_{k, n}(x) \leq \min \left\{\left(n \sin ^{2} \frac{\pi x_{k}}{p_{k}}\right)^{-1}, n\right\} .
$$

Let $I$ be a generalized interval containing $x$ such that $I \subset x+G_{k}, I$ is a union of cosets of $G_{k+1}$ and

$$
\frac{p_{k}}{n}-1<\frac{\mu(I)}{\mu\left(G_{k+1}\right)} \leq \frac{p_{k}}{n} .
$$

(Note that $p_{k} / n \geq 2$ since $n \leq 2^{L_{k}-1}$.) For $j=1,2, \ldots$, let $3^{j+1} I=3\left(3^{j} I\right)$, and $J=\min \left\{j \geq 1: 3^{j} I=x+G_{k}\right\}$. Then

$$
\begin{aligned}
\left|\sigma_{k, n} f(x)\right| \leq & \frac{1}{\mu\left(G_{k}\right)} \int_{3 I}|f(t)| K_{k, n}(x-t) d \mu(t) \\
& \quad+\sum_{j=1}^{J-1} \frac{1}{\mu\left(G_{k}\right)} \int_{3^{j+1} I \backslash 3^{j} I}|f(t)| K_{k, n}(x-t) d \mu(t) .
\end{aligned}
$$


By (3.4),

$$
\frac{1}{\mu\left(G_{k}\right)} \int_{3 I}|f(t)| K_{k, n}(x-t) d \mu(t) \leq \frac{3 \mu(I)}{\mu\left(G_{k}\right)} n \frac{1}{\mu(3 I)} \int_{3 I}|f(t)| d \mu(t) \leq C M f(x) .
$$

To estimate the last term in (3.5), we apply the other estimate in (3.4). We observe that when $x \in I, t \notin 3^{j} I, j=1, \ldots, J-1$,

$$
\left|\sin \frac{\pi\left(x_{k}-t_{k}\right)}{p_{k}}\right| \geq C \mu\left(3^{j-1} I\right) / \mu\left(G_{k}\right) .
$$

Hence

$$
\begin{aligned}
& \frac{1}{\mu\left(G_{k}\right)} \int_{3^{j+1} I \backslash 3^{j} I}|f(t)| K_{k, n}(x-t) d \mu(t) \\
& \leq \frac{\mu\left(3^{j+1} I\right)}{\mu\left(G_{k}\right)} \frac{1}{\mu\left(3^{j+1} I\right)} \int_{3^{j+1} I \backslash 3^{j} I}|f(t)|\left(n \sin ^{2} \frac{\pi\left(x_{k}-t_{k}\right)}{p_{k}}\right)^{-1} d \mu(t) \\
& \quad \leq C \frac{\mu\left(3^{j+1} I\right)}{\mu\left(G_{k}\right)} \frac{1}{n}\left[\frac{\mu\left(G_{k}\right)}{\mu\left(3^{j-1} I\right)}\right]^{2} \frac{1}{\mu\left(3^{j+1} I\right)} \int_{3^{j+1} I}|f(t)| d \mu(t) \\
& \leq C 3^{-j} \frac{\mu\left(G_{k}\right)}{n \mu(I)} M f(x) \\
& \leq C 3^{-j} M f(x) .
\end{aligned}
$$

Substituting these estimates into (3.5) and summing over $j$, we obtain (3.3). The lemma follows.

This completes the proof of the theorem.

\section{REFERENCES}

1. D.L. Burkholder, Distribution function inequalities for martingales, Ann. Probab. 1 (1973), 19-42. MR 51:1944

2. P. Simon, On the concept of a conjugate function, Colloq. Math. Soc. J. Bolyai, Fourier Analysis and Approximation Theory, 197619 (1978), 747-755. MR 81b:42084

3. P. Simon, On a maximal function, Ann. Univ. Sci. Budapest. Eötvös Sect. Math. 21 (1978), 41-44. MR 81b:42083

4. W.-S. Young, Mean convergence of generalized Walsh-Fourier series, Trans. Amer. Math. Soc. 218 (1976), 311-320. MR 52:14828

5. W.-S. Young, Almost everywhere convergence of Vilenkin-Fourier series of $H^{1}$-functions, Proc. Amer. Math. Soc. 108 (1990), 433-441. MR 90g:42057

6. W.-S. Young, Littlewood-Paley and multiplier theorems for Vilenkin-Fourier series, Canad. J. Math. 46 (1994), 662-672. MR 95c:42031

Department of Mathematics, University of Alberta, Edmonton, Alberta, Canada T6G 2G1 